\title{
Stable transmission characteristics of double-hump solitons for the coupled Manakov equations in fiber lasers
}

\section{Guoli Ma}

Beijing University of Posts and Telecommunications

\section{Qin Zhou}

Hubei Polytechnic University

\section{Weitian Yu}

Beijing University of Posts and Telecommunications

\section{Anjan Biswas}

Alabama A\&M University

Wenjun Liu ( $\square$ jungliu@bupt.edu.cn )

Beijing University of Posts and Telecommunications https://orcid.org/0000-0001-9380-2990

\section{Research Article}

Keywords: Double-hump solitons, soliton solution, coupled Manakov equations, fiber laser

Posted Date: September 3rd, 2021

DOl: https://doi.org/10.21203/rs.3.rs-862568/v1

License: (c) (1) This work is licensed under a Creative Commons Attribution 4.0 International License. Read Full License

Version of Record: A version of this preprint was published at Nonlinear Dynamics on September 28th, 2021. See the published version at https://doi.org/10.1007/s11071-021-06919-w. 
Noname manuscript No.

(will be inserted by the editor)

\title{
Stable transmission characteristics of double-hump solitons for the coupled Manakov equations in fiber lasers
}

\author{
Guoli Ma • Qin Zhou • Weitian Yu • \\ Anjan Biswas . Wenjun Liu
}

Received: date / Accepted: date

\begin{abstract}
The fiber laser has become an ideal ultrashort pulse source because of its cheap structure, high integration, convenient and controllable output direction, which greatly promotes the development and application of ultrafast optics. This paper mainly focuses on the control and amplification of doublehump solitons in fiber lasers theoretically. The bilinear forms and soliton solu-
\end{abstract}

Guoli Ma, Weitian Yu, Wenjun Liu

State Key Laboratory of Information Photonics and Optical Communications, and School of Science, P. O. Box 122, Beijing University of Posts and Telecommunications, Beijing 100876, China

Corresponding author: jungliu@bupt.edu.cn (Wenjun Liu)

Guoli Ma

Institute of Aeronautical Engineering, Binzhou University, Binzhou 256603, China

Qin Zhou

School of Mathematics and Physics, Hubei Polytechnic University, Huangshi 435003, China

Anjan Biswas

Department of Physics, Chemistry and Mathematics, Alabama A \& M University, Normal, AL 35762-4900, USA

Anjan Biswas

Mathematical Modeling and Applied Computation (MMAC) Research Group, Department of Mathematics, King Abdulaziz University, Jeddah-21589, Saudi Arabia

Anjan Biswas

Department of Applied Mathematics, National Research Nuclear University, 31 Kashirskoe Hwy, Moscow-115409, Russian Federation

Anjan Biswas

Department of Mathematics and Applied Mathematics, Sefako Makgatho Health Sciences University, Medunsa-0204, Pretoria, South Africa 
tions of the coupled Manakov equations are presented, and the transmission of double-hump solitons is discussed. The factors affecting the stable transmission of double-hump solitons are analyzed. The relevant conclusions have important guiding significance for understanding the generation of stable double-hump solitons in fiber lasers.

Keywords Double-hump solitons · soliton solution · coupled Manakov equations · fiber laser

\section{Introduction}

Optical soliton is one of the most studied form of solitons [1]. It not only can be conformed to the characteristics predicted by soliton theory [2-4], but also can be used as an optical information carrier for communication systems [5-7]. Up to now, optical fiber communication is one of the largest potential applications of optical solitons [8]. Optical fiber is the best carrier for transmitting optical signals, because it can confine the optical field to a plane perpendicular to the axis of the fiber core $[9,10]$.

In communications, the transformation content is achieved by expanding the range of carrier frequency in the form of bandwidth. In a linear optical fiber communication system, dispersion and nonlinear effects are highly destructive to the high-bit and long-distance transmission of the communication system. However, in the soliton optical fiber communication system, it is through the use of these two effects to solve these two problems at the same time [11]. The advantage of optical soliton communication is all-optical communication, and the signal does not need to be transferred to the electrical field for processing. Apart from being used in the communications field, optical solitons can also be used to construct optical switches. The advantage of optical soliton communication is that it does not change its shape when it interacts with other pulses $[12,13]$.

An excellent system for generating and observing solitons is the fiber laser [1]. In optical fibers, dispersion and nonlinearity play a key role in the properties of soliton fiber lasers [14]. Nonlinearity here specifically refers to the Kerr effect, that is, the refractive index of optical fibers has the nonlinear relationship with the pulse propagation intensity, and the phase of the pulse will also change with time $[15,16]$. Also, the optical fiber refractive index is caused by the third-order nonlinear susceptibility. Dispersion here refers to group velocity dispersion (GVD), that is, the refractive index is related to the wavelength, which causes the pulse to broaden in the time domain $[17,18]$.

In this paper, the effects of dispersion and nonlinearity on double-hump solitons will be investigated analytically. Double-hump soliton transmission in fiber lasers is expressed as the variable coefficient coupled Manakov equa- 
tions [19],

$$
\begin{aligned}
& i u_{x}+\beta_{2}(x) u_{t t}+2 \gamma(x)\left(|u|^{2}+|v|^{2}\right) u=0 \\
& i v_{x}+\beta_{2}(x) v_{t t}+2 \gamma(x)\left(|v|^{2}+|u|^{2}\right) v=0
\end{aligned}
$$

Here, $u$ and $v$ represent the pulse intensity, which are related to $x$ and $t$. $\beta_{2}(x)$ is the coeffiecient of GVD, and $\gamma(x)$ represents the nonlinearity. For Eq. (1), interactions between solitons have been studied [19-21]. However, the stable transmission characteristics of double-hump solitons in fiber lasers have not been reported before, which plays a key role in signal transmission and amplification in communication systems.

The generation and amplification of double-hump solitons will be investigated in the fiber lasers theoretically. The double-hump soliton solutions will be obtained in the coupled Manakov equations, which can model the doublehump soliton transmission in the fiber lasers in section 2 . We will analyze the factors affecting the stable transmission of double-hump solitons in section 3 . Results of this paper will be summarized at last.

\section{Double-hump soliton solutions}

At first, we will get the analytic double-hump soliton solutions with the Hirota method. We assume the rational transformation [22],

$$
u=p / h, \quad v=q / h .
$$

Here, $p, q$ and $h$ are all functions related to $x$ and $t$. We substitute transformation (2) into Eq. (1), and extract the coefficients of the same power of $h$, Eq. (1) can be divided into the following bilinear forms,

$$
\begin{array}{r}
i D_{x} p \cdot h+\beta(x) D_{t}^{2} p \cdot h=0, \\
i D_{x} q \cdot h+\beta(x) D_{t}^{2} q \cdot h=0, \\
\beta_{2}(x) D_{t}^{2} h \cdot h=2 \gamma(x)\left(|p|^{2}+|q|^{2}\right) .
\end{array}
$$

Here, $D_{x}$ and $D_{t}$ are defined as the Hirota operators [22].

Then, to obtain the double-hump soliton solution, we can assume

$$
p=\varepsilon p_{1}+\varepsilon^{3} p_{3}, \quad q=\varepsilon q_{1}+\varepsilon^{3} q_{3}, \quad h=1+\varepsilon^{2} h_{2}+\varepsilon^{4} h_{4},
$$

where

$$
\begin{aligned}
& p_{1}=\alpha_{1} e^{\delta_{1}}, \quad q_{1}=\alpha_{2} e^{\delta_{2}}, \quad p_{3}=\alpha_{3} e^{\delta_{1}+\delta_{2}+\delta_{2}^{*}} \\
& q_{3}=\alpha_{4} e^{\delta_{2}+\delta_{1}+\delta_{1}^{*}}, \quad h_{2}=\alpha_{5} e^{\delta_{1}+\delta_{1}^{*}}+\alpha_{6} e^{\delta_{2}+\delta_{2}^{*}}, \quad h_{4}=\alpha_{7} e^{\delta_{1}+\delta_{2}+\delta_{2}+\delta_{2}^{*}}
\end{aligned}
$$

with

$$
\delta_{j}(x, t)=\eta_{j}(x)+\sigma_{j} t \quad(j=1,2),
$$


and the asterisk represent the complex conjugate. We substitute expressions (4) into Eq. (3), and can obtain that

$$
\begin{aligned}
& \gamma(x)=\beta_{2}(x), \quad \eta_{j}(x)=i \sigma_{j}^{2} \int \beta_{2}(x) d x \\
& \alpha_{3}=\frac{\alpha_{1}\left|\alpha_{2}\right|^{2}\left(\sigma_{1}-\sigma_{2}\right)}{\left(\sigma_{1}+\sigma_{2}^{*}\right)\left(\sigma_{2}+\sigma_{2}^{*}\right)^{2}}, \quad \alpha_{4}=\frac{\alpha_{2}\left|\alpha_{1}\right|^{2}\left(\sigma_{2}-\sigma_{1}\right)}{\left(\sigma_{2}+\sigma_{1}^{*}\right)\left(\sigma_{1}+\sigma_{1}^{*}\right)^{2}} \\
& \alpha_{5}=\frac{\left|\alpha_{1}\right|^{2}}{\left(\sigma_{1}+\sigma_{1}^{*}\right)^{2}}, \quad \alpha_{6}=\frac{\left|\alpha_{2}\right|^{2}}{\left(\sigma_{2}+\sigma_{2}^{*}\right)^{2}}, \quad \alpha_{7}=\frac{\left|\alpha_{1}\right|^{2}\left|\alpha_{2}\right|^{2}\left|\sigma_{1}-\sigma_{2}\right|^{2}}{\left(\sigma_{1}+\sigma_{1}^{*}\right)^{2}\left(\sigma_{2}+\sigma_{2}^{*}\right)^{2}\left|\sigma_{2}+\sigma_{1}^{*}\right|^{2}} .
\end{aligned}
$$

Finally, we make $\varepsilon$ equal to 1 , and double-hump soliton solutions can present as

$$
\begin{aligned}
& q_{1}=\frac{p_{1}+p_{3}}{1+h_{2}+h_{4}}=\frac{\alpha_{1} e^{\delta_{1}}+\alpha_{3} e^{\delta_{1}+\delta_{2}+\delta_{2}^{*}}}{1+\alpha_{5} e^{\delta_{1}+\delta_{1}^{*}}+\alpha_{6} e^{\delta_{2}+\delta_{2}^{*}}+\alpha_{7} e^{\delta_{1}+\delta_{2}+\delta_{2}+\delta_{2}^{*}}}, \\
& q_{2}=\frac{q_{1}+q_{3}}{1+h_{2}+h_{4}}=\frac{\alpha_{2} e^{\delta_{2}}+\alpha_{4} e^{\delta_{2}+\delta_{1}+\delta_{1}^{*}}}{1+\alpha_{5} e^{\delta_{1}+\delta_{1}^{*}}+\alpha_{6} e^{\delta_{2}+\delta_{2}^{*}}+\alpha_{7} e^{\delta_{1}+\delta_{2}+\delta_{2}+\delta_{2}^{*}}} .
\end{aligned}
$$

\section{Discussion}
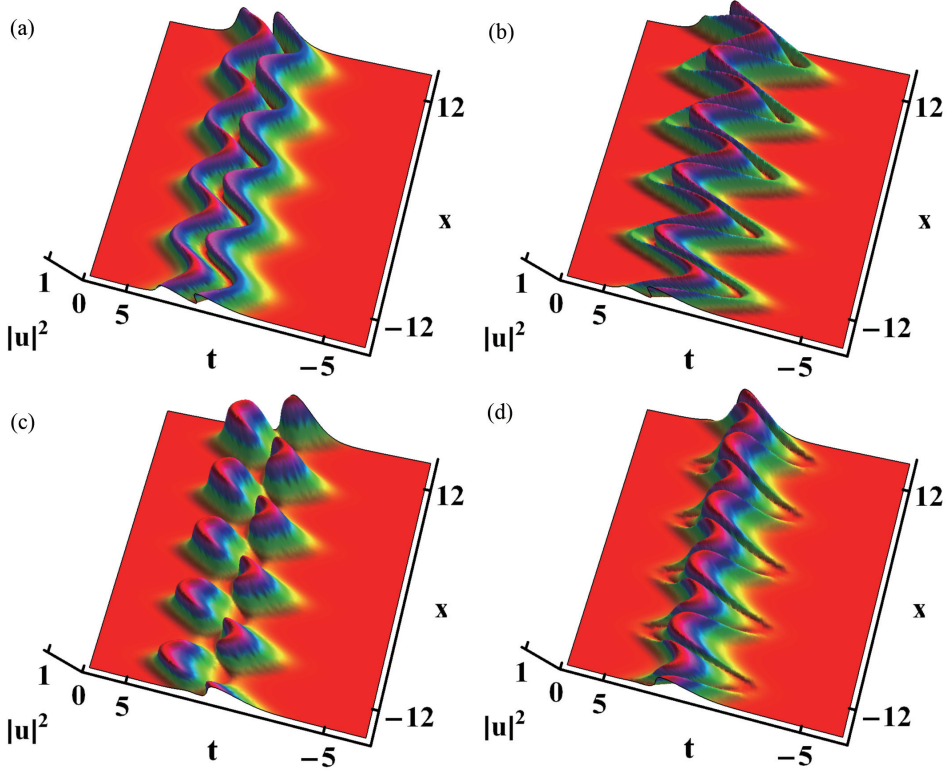

Fig. 1 Analysis of double-hump soliton transmission characteristics. The values of parameters in solutions (5) are: $\gamma(x)=\sin (x), \alpha_{1}=1+i, \alpha_{2}=1+i$ with (a) $\sigma_{1}=1+0.34 i$, $\sigma_{2}=2+0.44 i$; (b) $\sigma_{1}=1+0.81 i, \sigma_{2}=2+1.2 i$; (c) $\sigma_{1}=1-0.5 i, \sigma_{2}=2+0.28 i ;$ (d) $\sigma_{1}=1+0.38 i, \sigma_{2}=2+1.4 i$. 
For double-hump soliton solutions (5), we can choose different parameters to analyze the transmission characteristics of double-hump solitons. At first, we assume that $\gamma(x)=\beta_{2}(x)=\sin (x), \alpha_{1}=1+i, \alpha_{2}=1+i, \sigma_{1}=1+0.34 i$ and $\sigma_{2}=2+0.44 i$ in Fig. 1(a), and can observe double-hump solitons are generated. They show periodic transmission, and the spacing and speed between the two pulses remain unchanged during the transmission. The two pulses feel like forming a bound state, and the double-hump solitons also have particle properties. Changing the values of $\sigma_{1}$ and $\sigma_{2}$, the waveform of the pulse has changed, and the peak power of pulses is generated alternately in Fig. 1(b). However, the two pulses do not affect each other in the transmission process, and also show the periodic transmission of double-hump solitons. Pulses can be transformed into other local waves and appear alternately in Figs. 1(c) and $1(\mathrm{~d})$. No matter how their forms change, they do not affect each other and can maintain their independent characteristics. This is mainly due to the dispersion of the optical fiber is the sinusoidal profile in fiber lasers.
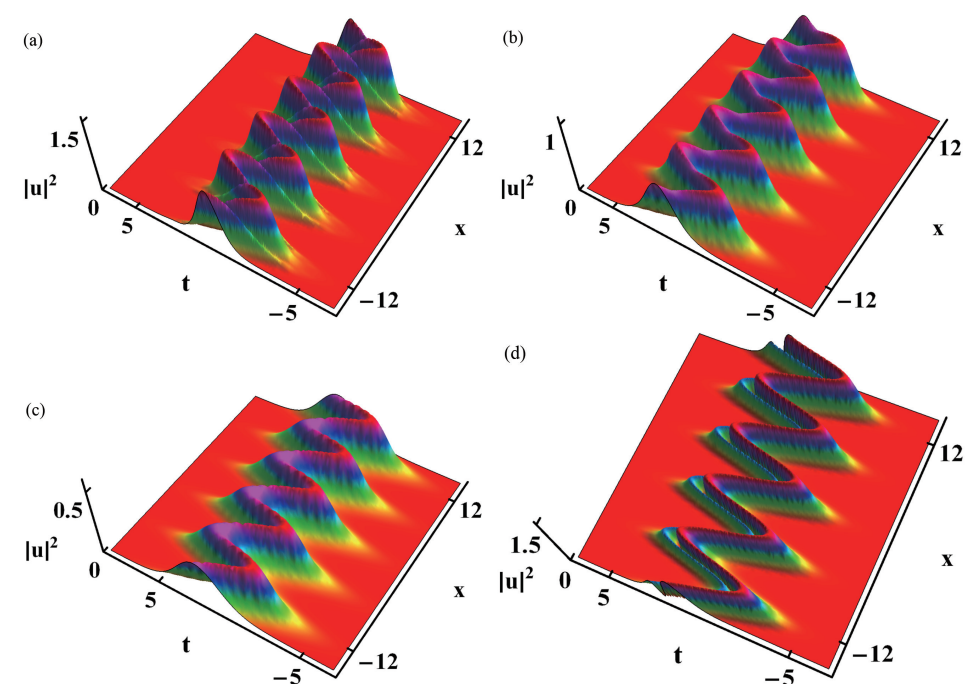

Fig. 2 Analysis of double-hump soliton transmission characteristics. The values of parameters in solutions (5) are: $\gamma(x)=\sin (x), \alpha_{1}=1+i, \alpha_{2}=1+i$ with (a) $\sigma_{1}=-1.1+i$, $\sigma_{2}=-1.3+2 i$; (b) $\sigma_{1}=0.9+i, \sigma_{2}=0.6+2 i$; (c) $\sigma_{1}=-0.6+i, \sigma_{2}=0.5+2 i$; (d) $\sigma_{1}=-1+i, \sigma_{2}=4+i$.

In Fig. 1, we mainly change the imaginary parts of $\sigma_{1}$ and $\sigma_{2}$ to realize different types of soliton transmission. Next, we will discuss how to change the real parts of $\sigma_{1}$ and $\sigma_{2}$ to realize the periodic transmission of double-hump solitons. In Fig. 2(a), $\sigma_{1}=1+0.34 i$ and $\sigma_{2}=2+0.44 i$, double-hump solitons exhibit periodic transmission, and have two obvious peaks. We reduce the values of the corresponding real parts of $\sigma_{1}$ and $\sigma_{2}$ in Fig. 2(b), and the peak of double-hump solitons are weakened. 
If we continue to reduce the value of the real part of $\sigma_{1}$ and $\sigma_{2}$, the strength of the double-hump soliton basically does not change in the transmission process in Fig. 2(c), which is similar to the traditional single soliton. If we set the value of the imaginary part to be equal, but the value of the real part is not equal, the double-hump soliton can realize non-interaction transmission. In the transmission process, the soliton strengths are not equal, but they do not affect each other in Fig. 2(d). Due to the same variation period, the soliton velocities are equal.
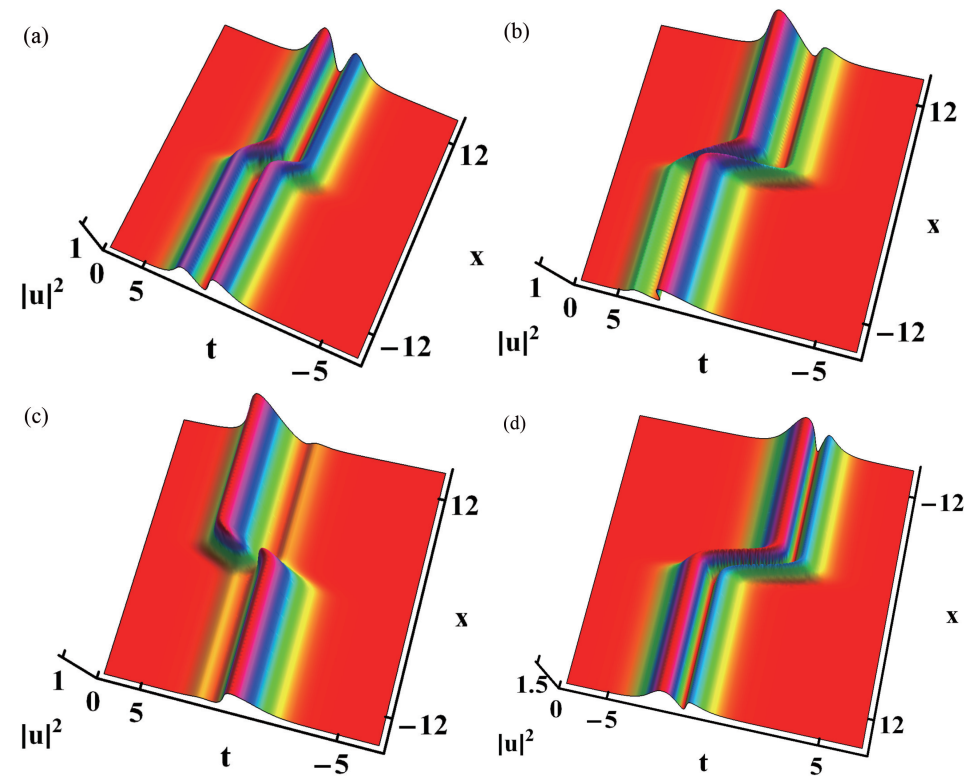

Fig. 3 Analysis of double-hump soliton transmission characteristics. The values of parameters in solutions (5) are: $\gamma(x)=-\exp \left(-x^{2}\right), \alpha_{1}=1+i, \alpha_{2}=1+i$ with (a) $\sigma_{1}=1+0.34 i$, $\sigma_{2}=2+0.44 i$; (b) $\sigma_{1}=1+0.81 i, \sigma_{2}=2+1.2 i$; (c) $\sigma_{1}=1-0.5 i, \sigma_{2}=2+0.28 i$; (d) $\sigma_{1}=-1+i, \sigma_{2}=4+i$.

Compared with Fig. 1, we assume the GVD function $\beta_{2}(x)$ is the Gaussian profile, that is $\beta_{2}(x)=\gamma(x)=-\exp \left(-x^{2}\right)$, double-hump solitons exhibit stable transmission. The same spacing is always maintained during transmission without interaction in Fig. 3(a). By changing the values of $\sigma_{1}$ and $\sigma_{2}$, some interaction transmission of double-hump solitons can be realized in Figs. 3(b)3(d). The pulse intensities exchange with each other in Figs. 3(b) and 3(c). However, when $\sigma_{1}=-1+i$ and $\sigma_{2}=4+i$, the pulse maintains its original strength during the transmission in Fig. 3(d), which is beneficial to improve the communication capacity of the system by reducing the pulse spacing.

When the GVD function $\beta_{2}(x)$ is the constant, such as $\beta_{2}(x)=\gamma(x)=-5$, double-hump solitons exhibit excellent transmission characteristics when $\sigma_{1}=$ 1 and $\sigma_{2}=2$ in Fig. 4(a). Double-hump soliton transmission is stable, and 


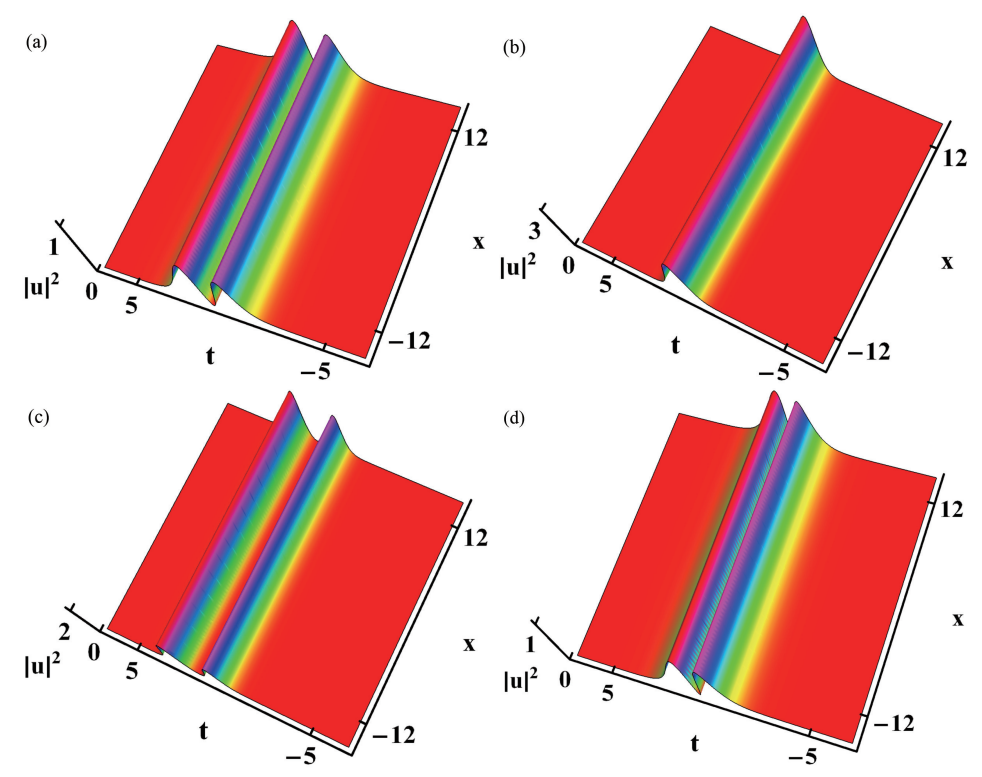

Fig. 4 Analysis of double-hump soliton transmission characteristics. The values of parameters in solutions (5) are: $\gamma(x)=-5, \alpha_{1}=1+i, \alpha_{2}=1+i$ with (a) $\sigma_{1}=1, \sigma_{2}=2$; (b) $\sigma_{1}=2, \sigma_{2}=1 ;$ (c) $\sigma_{1}=1.9, \sigma_{2}=2 ;$ (d) $\sigma_{1}=1, \sigma_{2}=4$.

there is no timing jitter. When $\sigma_{1}=2$ and $\sigma_{2}=1$, double-hump solitons merge into traditional solitons in Fig. 4(b). The strength of the soliton increases, and other properties remain unchanged. We can also change the soliton spacing by increasing or decreasing the values of $\sigma_{1}$ and $\sigma_{2}$ in Figs. 4(c) and $4(\mathrm{~d})$. No matter how small their spacing is, double-hump solitons can achieve the stable transmission. Those results are beneficial to the generation of doublehump solitons in fiber lasers. Furthermore, it is conducive to amplify the pulses in the fiber lasers.

\section{Conclusion}

The generation and transmission of stable double-hump solitons have been studied. Double-hump soliton solutions of the variable coefficient coupled Manakov equations have been obtained with the help of the Hirota method. When the GVD function is the sinusoidal function, the double-hump soliton has shown the periodic transmission. The corresponding transmission is related to the values of $\sigma_{1}$ and $\sigma_{2}$. The imaginary parts of them have affected the waveform and spacing of double-hump solitons. The real parts of them have affected the double-hump soliton strength. When the GVD function is the Gaussian function, the double-hump soliton has presented the stable transmission. Through adjusting the corresponding parameters, the purpose of soliton energy exchange has been realized. Finally, when the GVD function is con- 
stant, the stable double-hump soliton has been generated, and the soliton amplification has been demonstrated. Results in this paper is of great significance for the generation and amplification of double-hump solitons in fiber lasers.

\section{Acknowledgements}

We acknowledge the financial support from the National Natural Science Foundation of China (grant 11875009, 12075034); Fundamental Research Funds for the Central Universities (grant 2019XD-A09-3).

\section{Declarations}

Conflict of interest The authors declare that they have no conflict of interest concerning the publication of this manuscript.

Ethical approval The authors declare that they have adhered to the ethical standards of research execution.

Availability of data and material The authors declare that all data generated or analysed during this study are included in this article.

\section{References}

1. Agrawal, G. P.: Nonlinear Fiber Optics (6th Edition). Academic, Pittsburgh (2019)

2. Wazwaz, A.M.: Two new Painleve integrable KdV-Calogero-Bogoyavlenskii-Schiff (KdVCBS) equation and new negative-order KdV-CBS equation. Nonlinear Dyn. 104(4), 43114315 (2021)

3. Wang, L.L., Luan, Z.T., Zhou, Q., Biswas, A., Alzahrani, A.K., Liu, W.J.: Effects of dispersion terms on optical soliton propagation in a lossy fiber system. Nonlinear Dyn. 104(1), 629-637 (2021)

4. Yu, W.T., Zhang, H.X., Zhou, Q., Biswas, A., Alzahrani, A.K., Liu, W.J.: The mixed interaction of localized, breather, exploding and solitary wave for the $(3+1)$-dimensional Kadomtsev-Petviashvili equation in fluid dynamics. Nonlinear Dyn. 100(2), 1611-1619 (2020)

5. Chen, Z.M., Zeng, J.H.: Two-dimensional optical gap solitons and vortices in a coherent atomic ensemble loaded on optical lattices. Commun. Nonlinear Sci. Numer. Simul. 102 $105911(2021)$

6. Gnauck, A.H., Winzer, P.J.: Optical phase-shft-keyed transmission. J. Lightwave Technol. 23(1), 115-130 (2005)

7. Kippenberg, T.J., Gaeta, A.L., Lipson, M., Gorodetsky, M.L.: Dissipative Kerr solitons in optical microresonators. Science 361, 6402 (2018)

8. Turitsyn, S.K., Bale, B.G., Fedoruk, M.P.: Dispersion-managed solitons in fibre systems and lasers. Phys. Rep. 521(4), 135-203 (2012)

9. Becker, C., Stellmer, S., Soltan-Panahi, P., Dorscher, S., Baumert, M., Richter, E.M., Kronjager, J., Bongs, K., Sengstock, K.: Oscillations and interactions of dark and darkbright solitons in Bose-Einstein condensates. Nat. Phys. 4(6), 496-501 (2008)

10. Marin-Palomo, P., Kemal, J.N., Karpov, M., Kordts, A., Pfeifle, J., Pfeiffer, M.H., Trocha, P., Wolf, S., Brasch, V., Anderson, M.H., Rosenberger, R., Vijayan, K., Freude, W., Kippenberg, T.J., Koos, C.: Microresonator-based solitons for massively parallel coherent optical communications. Nature 546(7657), 274-279 (2017) 
11. Temprana, E., Myslivets, E., Kuo, B.P., Liu, L., Ataie, V., Alic, N., Radic, S.: Overcoming Kerr-induced capacity limit in optical fiber transmission. Science 348(6242), 1445-1448 (2015)

12. Li, B.J., Ozdemir, S.K., Xu, X.W., Zhang, L., Kuang, L.M., Jing, H.: Nonreciprocal optical solitons in a spinning Kerr resonator. Phys. Rev. A 103(5), 053522 (2021)

13. Huang, J.Y., Li, Q., Jian, Z.Y., Wai, P.A., Nakkeeran, K.: Combination and compression of multiple pulses with same or different wavelengths. J. Lightwave Technol. 38(24), 6932$6938(2020)$

14. Liu, W., Yang, C., Liu, M., Yu, W., Zhang, Y., Lei, M.: Effect of high-order dispersion on three-soliton interactions for the variable-coefficients Hirota equation. Phys. Rev. E 96(4), $042201(2017)$

15. Wang, Y.R., Hou, S.D., Yu, Y., Liu, W.J., Yan, P.G., Yang, J.B.: Photonic device combined optical microfiber coupler with saturable-absorption materials and its application in mode-locked fiber laser. Opt. Express 29(13), 20526-20534 (2021)

16. Yan, Y.Y., Liu, W.J.: Soliton rectangular pulses and bound states in a dissipative system modeled by the variable-coefficients complex cubic-quintic Ginzburg-Landau equation. Chin. Phys. Lett. 38(9), 094201 (2021)

17. Liu, W.J., Liu, M.L., Lin, S., Liu, J.C., Lei, M., Wu, H., Dai, C.Q., Wei, Z.Y.: Synthesis of high quality silver nanowires and their applications in ultrafast photonics. Opt. Express 27(12), 16440-16448 (2019)

18. Wang, L.L., Liu, W.J.: Stable soliton propagation in a coupled $(2+1)$ dimensional Ginzburg-Landau system. Chin. Phys. B 29(7), 070502 (2020)

19. Yu, W.T., Zhang, H.X., Wazwaz, A.M., Liu, W.J.: The collision dynamics between double-hump solitons in two mode optical fibers. Results Phys. 28, 104618 (2021)

20. Qin, Y.H., Zhao, L.C., Ling, L.M.: Nondegenerate bound-state solitons in multicomponent Bose-Einstein condensates. Phys. Rev. E 100, 022212 (2019)

21. Stalin, S., Ramakrishnan, R., Senthilvelan, M., Lakshmanan, M.: Nondegenerate solitons in Manakov system. Phys. Rev. Lett. 122, 043901 (2019)

22. Hirota, R.: Exact envelope-soliton solutions of a nonlinear wave equation. J. Math. Phys. 14(7), 805-809 (1973) 